This is author accepted manuscript, pre-copyedit version of an article published in:

Polymer Bulletin, Volume 77, issue 3, March 2020, Pages 1377 - 1397

DOI: https://doi.org/10.1007/s00289-019-02805-z

\title{
Kinetic model of a Diels-Alder reaction in a molten state: thermal and viscoelastic behaviour
}

Aleš Ručigaj, Tjaž Kobal, Urška Šebenik, Matjaž Krajnc

University of Ljubljana, Faculty of Chemistry and Chemical Technology, Večna pot 113, SI-1000 Ljubljana

E-mail: matjaz.krajnc@ fkkt.uni-lj.si

Phone: +38614798600

*Corresponding author

\begin{abstract}
Information on the reaction kinetics in the melt is a valuable prerequisite for a successful application of self-healing ability to benzoxazine-based thermosetting resins. The goal of this research was to develop a kinetic model of the Diels-Alder (DA) reaction in a molten state between $N$-phenylmaleimide (PMI) and benzoxazine containing furan group (G-f). Rheological data were found to be of significant importance in the kinetic model development and interpretation. The increase of viscosity, the occurrence of side reactions and equilibrium between DA and r-DA reaction were identified as possible reasons for the deceleration of reaction rate and limiting conversion. After a thorough investigation of the studied system, accompanied by the knowledge from the literature, the potential reason for the incomplete conversion of reactants was reduced to the crossover between chemically and diffusioncontrolled reaction due to the increasing viscosity of reaction system. Accordingly, the kinetic model based on the combination of Rabinowitch equation and Chern-Poehlein model was used to accurately describe the progress of the studied reaction in the molten state.
\end{abstract}

Keywords Diels-Alder reaction, Molten state, Thermal behaviour, Rheology, Kinetic model

\section{Introduction}

The Diels-Alder (DA) reaction is one of the most useful reactions in organic synthesis [1], especially for forming 6-membered systems with good control over regio- and stereochemical properties [2]. This (4+2) cycloaddition between diene and dienophile is classified among the group of "click" reactions, which are known for their versatility, efficiency and selectivity $[3,4]$. The reaction leads to a mixture of two diastereomers called endo and exo adducts. DA reaction can be reversed via retro-Diels-Alder (r-DA) reaction at the appropriate temperature range, resulting in the primary diene and dienophile. As a result of this thermal reversibility, the combination of DA and r-DA reaction is commonly used in the synthesis of self-healing materials $[5,6]$, which are characterized by the capability of self-repairing or recovering after mechanically or thermally induced damages. Compounds incorporating furan and maleimide moieties are commonly used as dienes and dienophiles, respectively. The 
choice to use these particular systems arises from the fact that the furan/maleimide DA adducts display a relatively low temperature of decoupling through its r-DA reaction. In this study, $N$-phenylmaleimide (PMI) was used as a dienophile and benzoxazine containing furan group (G-f) as diene. Benzoxazine resins have a wide range of mechanical and physical properties that can be tailored to various needs. Bio-based benzoxazines can be synthesized readily by replacing synthetic starting materials from non-renewable sources with the natural ones [7, 8]. The use of bio-based renewable materials is preferable due to environmental issues and gradual depletion of non-renewable resources. By using benzoxazine containing furan group, the capability of self-healing can be incorporated in this novel type of thermosetting resins. The focus of this study was oriented towards the kinetics of DA reaction between PMI and G-f in a solventless molten state. So far, the majority of kinetic studies were focused on DA reaction between maleimide and furan functionalities in solution [9-12]. Nonetheless, only a few published studies exist where DA reaction was studied in the molten state [12-14] and no work has been done so far on describing the kinetics of DA reaction in the molten state.

Many examples describing reactions in the molten state were presented in the literature [15-18], however, the kinetics of reactions proceeding in a melt has not been extensively characterized. Reactions in a melt are believed to proceed more rapidly than those in a solid because the stabilizing forces of a crystalline structure are absent and there is greater stereochemical freedom [16]. When the heterogeneity and crystal order associated with the solid state is lost, it is expected that such reactions obey rate laws characteristic of homogeneous rate processes, which are controlled by reactant's concentrations [17]. Melt reactions are a subgroup of solventless reactions, which are regarded as a part of green chemistry approaches to the synthesis [19]. Performing a chemical synthesis in the absence of solvent means that the concentration of reactants will be at their maximum. In principle, this should lead to a faster reaction in comparison to a reaction in solution [15]. On the other hand, factors related to diffusion, heat transfer and mass transfer may have a great impact on the progress of the reaction [15]. In the literature, only poor information is available on the competing roles of the chemical reaction and diffusion in the melt. During the reaction, the viscosity of the reaction mixture increases due to greater molecular weight of products compared to reactants. Based on the fact that the melt's viscosity has an important impact on the mobility of reacting species, a major increment of viscosity can cause the rate of reaction to become controlled by diffusion of reactants. Crossover between the chemically and the diffusion controlled rate of reaction can be described with Rabinowitch equation [20], which allows the timescale of the reaction to be the sum of the timescale for the diffusion and the timescale of chemical reaction in the absence of diffusion. Diffusion-controlled reactions have been the subject of a considerable number of studies and several models have been proposed to relate the rate constant to the 
appropriate diffusion constant. The majority of models are semi-empirical since mechanistic models would involve many complex steps and a large number of adjustable parameters. The majority of semi-empirical models is based on the free-volume concept [21-25]. Free volume is a consequence of considerable looseness of packing in the structure of the liquid and can, therefore be defined as empty space between the molecules. This empty space is highly correlated to the viscosity of the medium since the free volume available for the movement of molecules decreases with increasing viscosity. When the free volume decreases below the critical value, movement of molecules becomes restricted and reaction diffusion-controlled.

As mentioned above, the focus of this paper is on the kinetic investigation of DA reaction between $N$ phenylmaleimide (PMI) and benzoxazine containing furan group (G-f) in the molten state. The progress of the reaction at different temperatures was compared. Almost the same final conversion was achieved at all reaction temperatures and reached the final value after approximately 50 minutes. Multiple possibilities were highlighted as possible reasons for deceleration of the reaction rate and limiting conversion as a consequence. Finally, after the systematic investigation of the studied system accompanied by the knowledge from the literature and additional experiments, the incomplete conversion of reactants was explained with the crossover between chemically and diffusion-controlled reaction. Following the research phenomenological kinetic model has been proposed, which allows for an accurate prediction of the progress of the reaction of PMI/ G-f system in a molten state over the whole course of the reaction.

\section{Experimental section}

\section{1. Materials}

Reactant $N$-phenylmaleimide (PMI, 97\%) was purchased from Aldrich, and reactant 3-(furan-2-ylmethyl)-8methoxy-3,4-dihydro-2H-benzo[e][1,3]oxazine (G-f) was synthesized according to the literature [26]. Aniline (99.5\%), acetic anhydride (98.5\%) and hexane were purchased by Merck. Guaiacol (98\%), furfurylamine (99\%), maleic anhydride $(97 \%)$ and paraformaldehyde were purchased from Aldrich, and deuterated chloroform $(99.8 \%$ D) was purchased from Euriso-top. All chemicals were used as purchased.

\section{2. Characterization}

The proceeding of the DA/rDA reaction was studied by ${ }^{1} \mathrm{H}$ NMR, differential scanning calorimetry (DSC) and rheological measurements of viscoelastic properties. Reactants were grounded in a mortar and mixed in a stoichiometric amount. 
DA/r-DA kinetic investigations were performed by isothermal heating of the PMI/G-f mixture at 60, 70, 80, 90, 100 and $110^{\circ} \mathrm{C}$. Each experiment was repeated in three parallels and average values were used in mathematical modelling of reaction. The progress of the reaction (conversion of reactant G-f) as a function of time was monitored by ${ }^{1} \mathrm{H}$ NMR analysis after the PMI/G-f reaction mixture sample was rapidly cooled to the ambient temperature by liquid nitrogen. The remained amount of G-f reactant was compared to the amount of DA adducts in the mixture, from which the conversion of the reaction was calculated. ${ }^{1} \mathrm{H}$ NMR analyses were performed on a Bruker Avance III $500 \mathrm{MHz}$ NMR spectrometer (Billerica, USA) at the temperature $296 \mathrm{~K}$ and frequency 500.13 MHz. Deuterated chloroform $\left(\mathrm{CDCl}_{3}\right)$ containing tetramethylsilane (TMS) as an internal standard was used as a solvent.

Calorimetric studies were carried out on Mettler Toledo DSC1 instrument with intra-cooler using STAR software (Greifensee, Switzerland). In and Zn standards were used for the temperature calibration and for the determination of the instrument time constant. Samples of around $10 \mathrm{mg}$ were weighed in standard $40 \mu \mathrm{L}$ aluminium pans. DSC measurements were performed in the nitrogen atmosphere with $30 \mathrm{~mL} / \mathrm{min}$ nitrogen flow rate. In order to observe the temperature dependent behaviour of the reaction mixture, non-isothermal experiments with heating and cooling runs were performed. The samples were heated from $20^{\circ} \mathrm{C}$ to $140{ }^{\circ} \mathrm{C}$ in the initial run, which was followed by a cooling run (from $140^{\circ} \mathrm{C}$ to $60^{\circ} \mathrm{C}$ or $20^{\circ} \mathrm{C}$ ) and a second heating run (from 20 or 60 to $140{ }^{\circ} \mathrm{C}$ ). A heating/cooling rate of $2 \mathrm{~K} / \mathrm{min}$ was used.

Viscoelastic properties of the melted reaction mixture were measured on Anton Paar Physica MCR 301 rheometer (Graz, Austria) equipped with $25 \mathrm{~mm}$ diameter plate-plate geometry. Isothermal experiments (at 60, 70, 80, 90, 100 and $110^{\circ} \mathrm{C}$ ) and dynamic experiments (from 60 to $140{ }^{\circ} \mathrm{C}$, using a heating/cooling rate of $2 \mathrm{~K} / \mathrm{min}$ to compare with the similar DSC experiments) were performed at $1 \mathrm{~Hz}$ in the range of linear viscoelastic behaviour using $1 \%$ deformation. The results are given only in the temperature regions where the measured torque did not surpass the maximum torque of the instrument.

\section{Results and discussion}

\subsection{Dynamic DSC and rheological characterization of the reaction system}

Isothermal experiments which were used for the investigation of the kinetics of Diels-Alder (DA) reaction in a molten state were designed on the basis of dynamic DSC analysis results. Dynamic DSC experiments (Figure 1) were conducted by heating and cooling the stoichiometric mixture of PMI and G-f in three consecutive runs as mentioned in the experimental part. The non-isothermal rheological measurements (Figure 2) were performed at 
equivalent temperature program as DSC measurements (from 60 to $140{ }^{\circ} \mathrm{C}$, using heating/cooling rate $2 \mathrm{~K} / \mathrm{min}$ ) in order to gain a systematic insight into the processes occurring in the reaction mixture.

When heating the reaction mixture under the conditions of the first temperature interval (Figure 1a, black line), the exothermic peak, appearing immediately after the melting process of reactants $\left(\sim 58^{\circ} \mathrm{C}\right)$, showed a maximum at around $70{ }^{\circ} \mathrm{C}$. The first exothermic peak was associated with the DA reaction, which was possible only after the melting of the PMI/G-f mixture. Note, the presence of DA/r-DA reaction in a solution was observed already at 30 ${ }^{\circ} \mathrm{C}[12]$. The exothermic peak of DA reaction lasted up to approximately $80{ }^{\circ} \mathrm{C}$, where the endothermic peak of $\mathrm{r}$ DA reaction of endo adducts appeared. According to the literature [27], the r-DA reaction of endo adduct has a significantly lower activation barrier compared to exo adduct and, therefore, occurs at the lower temperature. Following the r-DA reaction of endo adducts, exo adducts were formed by DA reaction which resulted in a weak exothermic peak with a maximum at around $100{ }^{\circ} \mathrm{C}$ (Figure 1). Thereafter, an endothermic peak, linked to r-DA reaction of exo adducts, lasted up to $140^{\circ} \mathrm{C}$, at which region also the onset of the polymerization might be detected. A more detailed description of the polymerization processes may be found elsewhere [12]. Throughout the whole cooling interval (from 140 to $60^{\circ} \mathrm{C}$ ), DSC thermogram (Figure 1, red curve) showed a broad peak of weak exothermic behaviour which monotonously decreases with decreasing temperature. Based on the fact that DA reaction is exothermic, the phenomenon could be explained by the fact that there is an equilibrium between DA and r-DA reaction (reactants are gradually transformed to DA-adducts while temperature decreases). No signal indicating crystallization was observed above $60^{\circ} \mathrm{C}$. DSC signals of the thermogram of the third temperature interval when the reaction mixture was once again heated to $140{ }^{\circ} \mathrm{C}$ at the heating rate $2 \mathrm{~K} / \mathrm{min}$ (Figure 1 , blue line), corresponded to signals observed in the thermogram of the first run above $60{ }^{\circ} \mathrm{C}$. However, the intensity of its signals was significantly lower, indicating a lower extent of DA reaction. To confirm the absence of crystallization and melting signal also at temperatures lower than $60{ }^{\circ} \mathrm{C}$ an additional DSC experiment (after the rapid cooling from $1^{\text {st }}$ run) was run in the range between 20 and $140{ }^{\circ} \mathrm{C}$ (Figure 1 , dashed line, $* 2^{\text {nd }}$ run). The reason for the absence of PMI/G-f mixture crystallization/melting is most likely the same as for the lower extent of DA reactions during the second heating run: the presence of DA bonds between PMI and G-f, which should result in increased viscosity and moduli of the investigated material. More detailed description of the thermal behaviour and endo/exo adducts temperature dependence based on DSC experiments were thoroughly described in one of our previous studies [12]. 


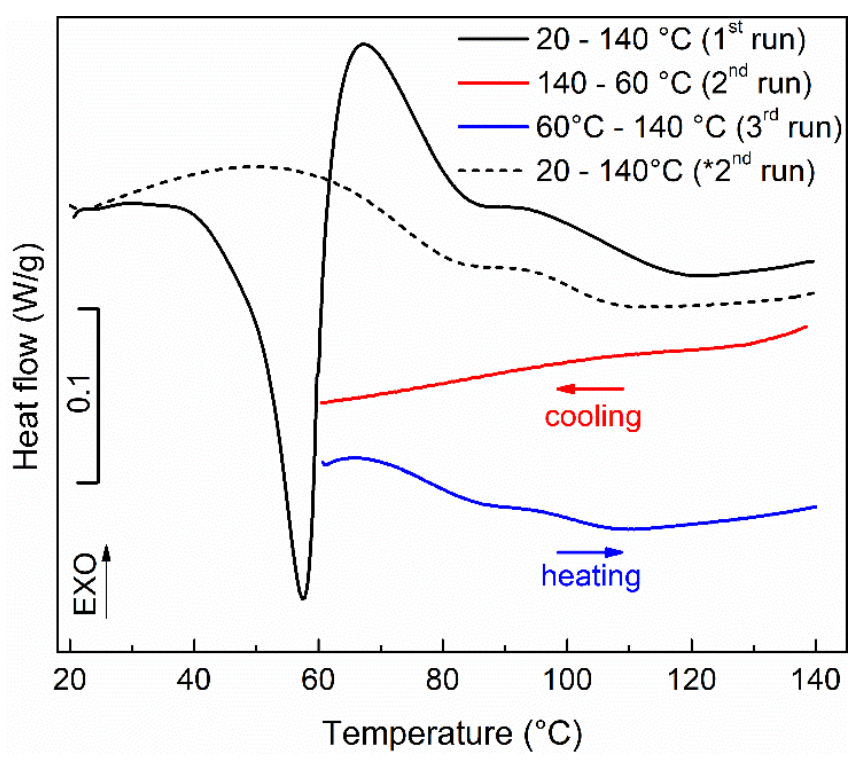

Fig. 1 DSC analyses of consecutive heating/cooling intervals for the stoichiometric mixture of PMI and G-f using $2 \mathrm{~K} / \mathrm{min}$ heating/cooling rate; first $\left(1^{\text {st }}\right)$ run from 20 to $140{ }^{\circ} \mathrm{C}$ (black), second $\left(2^{\text {nd }}\right)$ run from 140 to $60{ }^{\circ} \mathrm{C}$ (red), and third $\left(3^{\text {rd }}\right)$ run from 60 to $140{ }^{\circ} \mathrm{C}$ (blue) and; first run from 20 to $140{ }^{\circ} \mathrm{C}$ (black), rapid cooling and second $\left(* 2^{\text {nd }}\right)$ run from 20 to $140{ }^{\circ} \mathrm{C}$ (dashed).

Similar trends as in the case of DSC analyses were observed by the non-isothermal rheological experiments (Figure 2). The increase of the complex viscosity in the first temperature interval, which corresponds to the first exothermic peak of the first DSC run, with a peak value at around $70{ }^{\circ} \mathrm{C}$, was attributed to the formation of the DA adducts. The decrease of complex viscosity, which followed, is in line with endothermic DSC signal and corresponding to r-DA reaction. The consequence of an exo adduct formation (Figure 2a, black line) may be observed as well, as a change in complex viscosity curve slope at around $100^{\circ} \mathrm{C}$. Note, the decrease of the complex viscosity was, along with the r-DA reaction, also the result of the temperature effect (the higher the temperature, the lower the viscosity). On the other hand, inspecting rheological curves of the second (cooling) and third (heating) run (Figure 2a, red and blue line, respectively) and comparing the curve of the second heating run with that of the first heating (Figure 2a, black line) additionally proves the formation of DA adducts during cooling run. By cooling the reaction mixture back to $60^{\circ} \mathrm{C}$ the values of complex viscosity increased and reached a higher value than that of the reaction mixture in the initial state. During the second heating run, complex viscosity decreased due to r-DA reaction and viscosity temperature dependence. Moreover, the complex viscosity values above $125{ }^{\circ} \mathrm{C}$ were practically identical for all three runs, which confirmed the high extent of r-DA reaction and an insignificant viscosity increase due to polymerization, which might occur at these temperatures. 
In addition to the complex viscosity, storage ( $\left.\mathrm{G}^{\prime}\right)$ and loss modulus ( $\mathrm{G}^{\prime}$ ') as a function of temperature could be shown (Figure $2 b$ ) to inspect the viscoelastic properties of the melt. Higher loss modulus compared to storage modulus indicates a more liquid-like character of the system (Figure 2b). On the other hand, storage modulus (G') represents an elastic response of the system and its increase could be associated to processes of structure formation such as crystallization or chemical bonds formation (reaction). In presented system, chemical bonds were formed during the DA reaction and broken during r-DA reaction. Thus, the G' value was expected to increase in the range of DA reaction and decrease in the range of r-DA reaction.

The trend of fast increasing of G' (Figure 2b) with decreasing temperature in the second interval (cooling) indicates a potential cross-over of $\mathrm{G}^{\prime}$ and $\mathrm{G}^{\prime}$ ' at temperatures below $60^{\circ} \mathrm{C}$. Of course, such material properties cannot be measured by a rheometer, but rather a dynamic mechanical analysis should be used instead. However, the moduli values were of the same order of magnitude already at $60{ }^{\circ} \mathrm{C}$ (at the end and at the beginning of the second and third interval, respectively), however, a detailed investigation of mechanical properties is beyond the scope of this manuscript. A slight increase of $\mathrm{G}^{\prime}$ values was expected in the range of the exo DA adduct formation $\left(\sim 100{ }^{\circ} \mathrm{C}\right)$, but the G' values were already too low after r-DA reaction of endo adducts and the measured points dissipated too much.

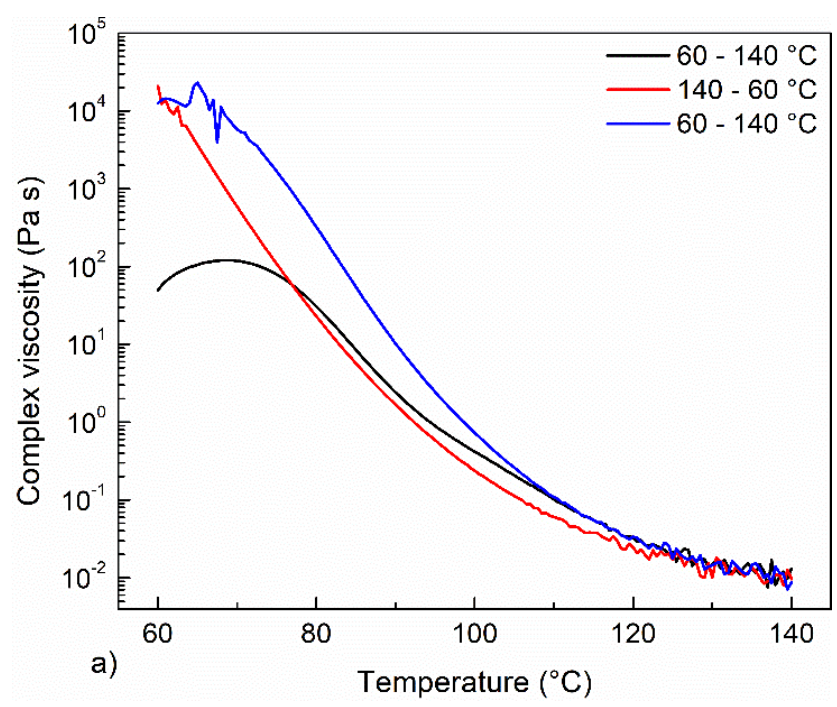




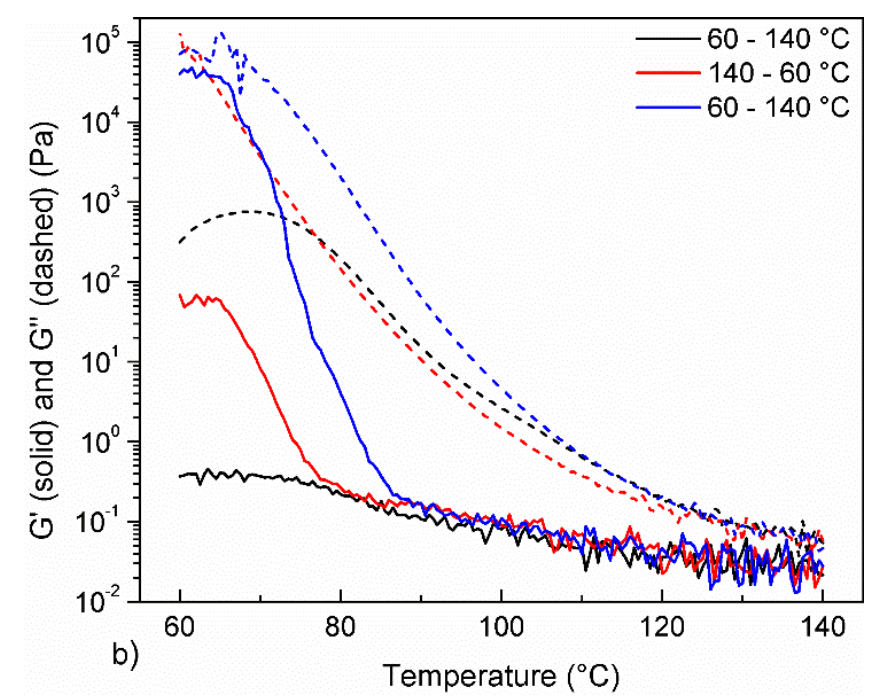

Fig. 2 Complex viscosity (a), storage modulus and loss modulus (b) as a function of temperature in consecutive heating and cooling $(2 \mathrm{~K} / \mathrm{min})$ of the reaction mixture.

On the basis of the above-obtained knowledge, the temperature range from 60 to $110{ }^{\circ} \mathrm{C}$ was estimated as the most appropriate for performing isothermal experiments for kinetics investigation. In such a way, the combined effect of DA and r-DA reactions at isothermal conditions could be studied.

\section{2. Isothermal experiments of stoichiometric mixture}

Based on the dynamic DSC analysis, DA reaction was investigated by isothermal heating of the stoichiometric mixtures of PMI and G-f in the range from $60{ }^{\circ} \mathrm{C}$ to $110^{\circ} \mathrm{C}$. The upper limit of the temperature range was defined by a predominant effect of r-DA reaction on the progress of reaction and probability of side reactions in the studied reaction system, and the lower limit by the melting of the reaction mixture. Based on the dynamic characterisation of the reaction system, the higher the temperature of the isothermal heating, the higher the effect of r-DA reaction at the increased concentration of DA adducts was expected. The progress of the reaction as a function of time was monitored by ${ }^{1} \mathrm{H}$ NMR. The results obtained by those experiments were thereafter used in the kinetic modelling of the DA reaction in a molten state. Isothermal rheological experiments in the same temperature range were performed (Figure 3). Rheological data was once again found to be of a significant value in the kinetic model development and interpretation.

The experimental results showed that the rate of reaction increased with increasing temperature (Figure $3 b$ ), although the same final conversion was achieved at 70 and $80{ }^{\circ} \mathrm{C}$ and reached the final value after 90 minutes (Figure 3a). At the temperatures of $100^{\circ} \mathrm{C}$ and $110^{\circ} \mathrm{C}$, as expected, the rate of reaction decreased compared to the 
isothermal experiments at lower temperatures (Figure 3a). Moreover, the final conversion, achieved in those experiments, was substantially lower, when compared to the final conversion obtained at lower temperatures and even started to decrease after prolonged heating. The possibility of unwanted side-reactions could not be completely eliminated for the experiment at $110^{\circ} \mathrm{C}$, however, minor effect, if any, was proved [12]. The major reason in decrease of conversion at 100 and $110^{\circ} \mathrm{C}$ was associated with the proceeding of r-DA reaction, which was previously indicated also by the dynamic DSC analysis and rheometry.

As expected, following the knowledge gained by performing non-isothermal DSC measurements, prolonged time of approximately 15 minutes was needed for sample melting at $60^{\circ} \mathrm{C}$. The progress of conversion as a function of time was sigmoidal (Figure 3b), which is characteristic for autocatalytic processes [17]. Apparent autocatalysis can be explained by increasing volume of melt, where the rate of reaction is greater compared to the rate of reaction in solid state [28]. Due to the fact that results showed the disproportionate influence of melting, data at this temperature cannot be completely trusted. Based on presented arguments, just one set of measurements was performed at the temperature of $60{ }^{\circ} \mathrm{C}$, and this isothermal experiment was not included in the development of the kinetic model of Diels-Alder reaction in a molten state.

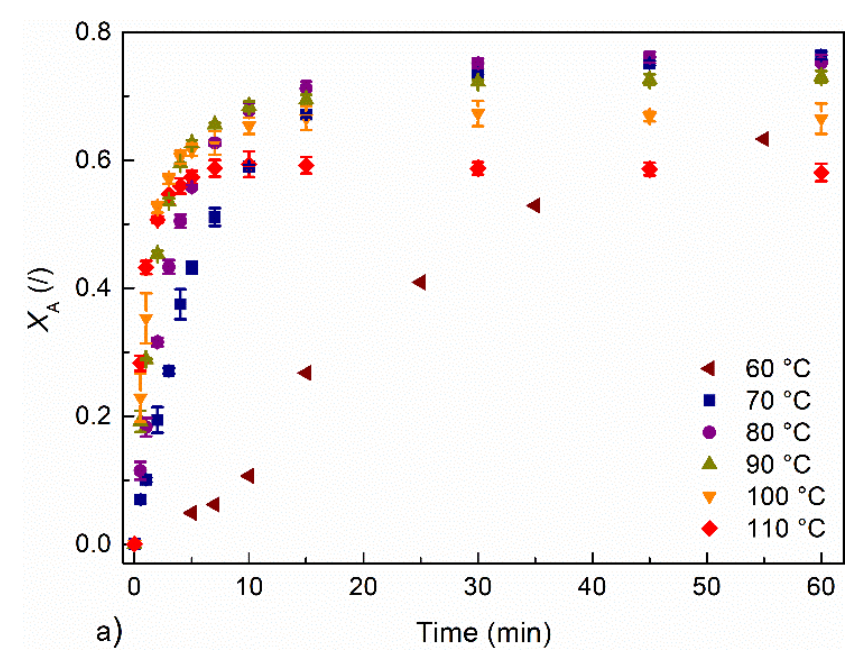




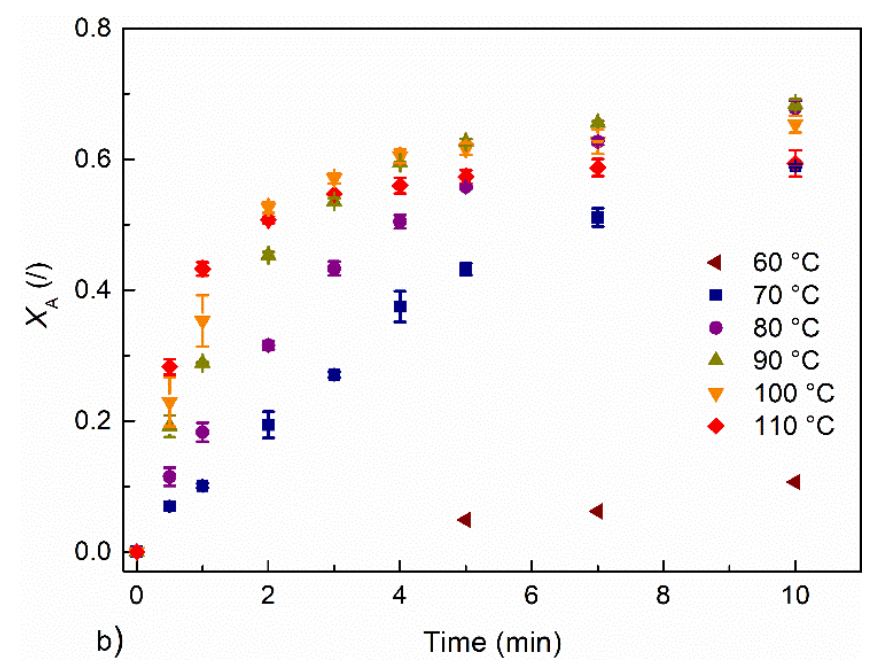

Fig. 3 Progress of conversion during the whole course of reaction (a) and during the first 10 minutes (b) for isothermal experiments at different temperatures. Error bars show standard deviation of experimental results. For $60{ }^{\circ} \mathrm{C}$ preliminary results showed profound influence of melting and, thus, just one set of experimental point was obtained.

Interestingly, the maximum conversion in the range from 70 to $90{ }^{\circ} \mathrm{C}$ did not change significantly and never reached the value of unity. Based on the fact that no significant change of final conversion with temperature was observed, the option of equilibrium between DA and r-DA reaction, as the main reason for the slowdown of reaction, was eliminated. Further investigation was, therefore, designed to shed light on processes that limit the rate of reaction and prevent the complete conversion of reactants. The increase of viscosity and occurrence of side reactions were identified as possible reasons for deceleration of reaction rate and limiting conversion.

The absence of side reactions was proved by ${ }^{1} \mathrm{H}$ NMR. Detailed ${ }^{1} \mathrm{H}$ NMR spectra of reactants (PMI and G-f) and products (endo and exo adducts) were already described by Štirn et. al. [12] In their work, it was shown that by the progress of the reaction, the intensity of characteristic peaks decreases. It is argued that in the case of unwanted side reaction a peak which was not identified as a characteristic peak of reactants or products of DA reaction would appear in the spectra during the progress of the reaction. Since the complete ${ }^{1} \mathrm{H}$ NMR could be assigned to molecules known to participate in DA reaction, the possibility of side reactions was eliminated.

After a thorough investigation of the studied system accompanied by the knowledge from the literature, the potential reason for the incomplete conversion of reactants was reduced to the crossover between chemically and diffusion-controlled reaction due to the increasing viscosity of reaction system. In order to conclude a possible assumption, the complex viscosity of melt was monitored during the reaction. The results presented in Figure 4 show the increase in complex viscosity by a few orders of magnitude during the whole course of the reaction. The 
increase of complex viscosity was greater at lower temperatures, which can be explained by the temperature dependence of viscosity. The diffusion coefficients of reactants decrease during the reaction, which consequently hinders the mobility of molecules. As conversion increases, a point is reached where the rate of reaction is no longer controlled by intrinsic reaction kinetics and, therefore, by the activation energy of the reaction, but by the rate of reactants' approach. Crossover between chemically and diffusion controlled rate of reaction can be described by Rabinowitch equation [29], which allows the timescale of the reaction to be the sum of the timescale for the diffusion plus the timescale of chemical reaction in the absence of diffusion.

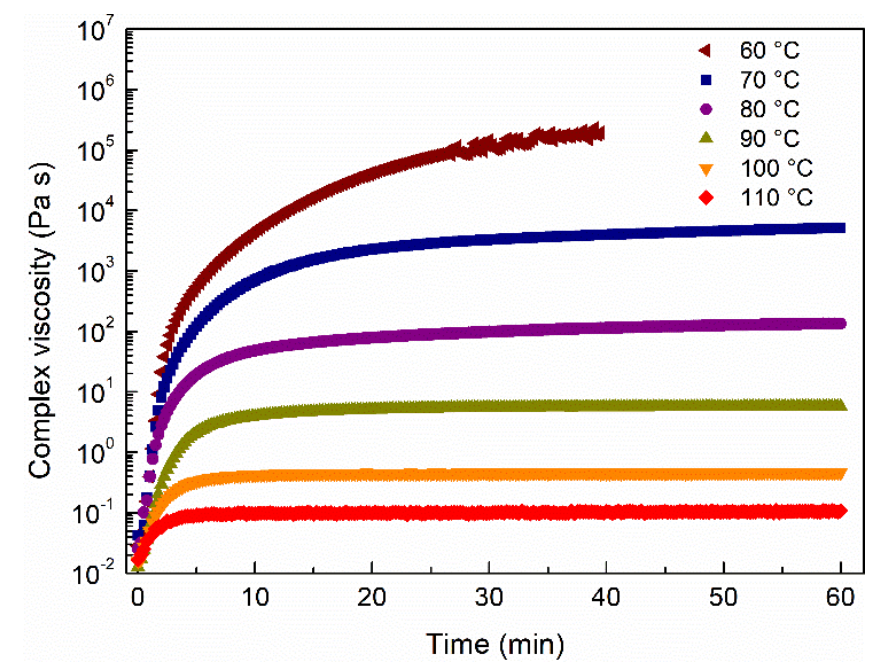

Fig. 4 Evolution of complex viscosity during the progress of reaction at different temperatures.

\section{3. Kinetic model of a Diels-Alder reaction in a molten state}

Based on the results of isothermal experiments with stoichiometric mixture supported by the rheological data it was concluded that in addition to the effect of DA and r-DA reaction on the rate of reaction, there is a transition from chemically to diffusion controlled reaction kinetics during the progress of reaction due to the increase in viscosity for a few orders of magnitude. In the following paragraphs, the derivation of kinetically controlled rate of reaction is shown in the first part, whereas in the second part, various models used to describe the crossover between chemically and diffusion-controlled reaction kinetics are presented and supplemented with the more meticulous derivation of the kinetic model proposed by Chern and Poehlein [21], which was used to describe the studied reaction system. Finally, kinetic parameters were derived from experimental data.

\section{Kinetically controlled rate of reaction}

Based on the experimental data, two assumptions were made in the development of the kinetic model. The first assumption was that according to the results of DSC and rheological experiments (Figure 1a), the dominancy of 
the effect of the DA reaction was observed in the range of $60-90^{\circ} \mathrm{C}$, whereas at $100{ }^{\circ} \mathrm{C}$ and $110{ }^{\circ} \mathrm{C}$ the effect of the rDA reactions started to prevail. The second assumption was that the mechanism of chemical reaction was considered to be unaffected by the reaction medium as also described accordingly by other authors [12]. Based on the latter assumption, the reaction was investigated as a bimolecular second-order reversible reaction. The simple reaction equation defining the progress of the reaction can, thus, be written as Eq. (1)

$$
\mathrm{A}+\mathrm{B} \leftrightarrow \mathrm{P}
$$

where symbol A stands for G-f. The conversion of reactants was determined by ${ }^{1} \mathrm{H}$ NMR as described in the experimental section. The symbol B is used for the second reactant in Diels-Alder reaction (PMI) and P for the products of DA reaction (sum of endo and exo-adducts). This type of denotation is used throughout the development of the kinetic model. The kinetics of the reaction could be described as a solvent-free homogeneous rate process, where the crystal order was lost in the completely melted reaction mixture. For that reason, the kinetic behaviour can be described in concentration terms $[17,18,28]$. The rate of the studied reaction in the molten state was consequently described as:

$$
\left(-r_{\mathrm{A}}\right)=-\frac{d c_{\mathrm{A}}}{d t}=k_{1} c_{\mathrm{A}} c_{\mathrm{B}}-k_{2} c_{\mathrm{P}}
$$

where $r_{\mathrm{A}}$ is the rate of reaction, $c_{\mathrm{A}}$ concentration of reactant $\mathrm{A}, c_{\mathrm{B}}$ concentration of reactant $\mathrm{B}, c_{\mathrm{P}}$ concentration of products $\mathrm{P}, k_{1}$ rate constant of DA reaction and $k_{2}$ rate constant of r-DA reaction. All experiments were performed with the stoichiometric mixture of reactants $\mathrm{A}$ in $\mathrm{B}, c_{\mathrm{A}}=c_{\mathrm{B}}$. Based on the initial concentration of reactant $\mathrm{A}\left(c_{\mathrm{A} 0}\right)$ and its conversion $\left(X_{\mathrm{A}}\right)$ the concentration of reactant $\mathrm{A}$ can be defined as:

$$
c_{\mathrm{A}}=c_{\mathrm{A} 0}\left(1-X_{\mathrm{A}}\right)
$$

Eq. (2) can also be re-written based on the conversion of reactant $\mathrm{A}\left(X_{\mathrm{A}}\right)$ :

$$
\left(-r_{A}^{*}\right)=\frac{d X_{\mathrm{A}}}{d t}=\underset{1 \times \mathrm{xA} 0}{k c}\left(1-X_{\mathrm{A}}\right)^{2}-k_{2}^{X} \text {. }
$$

In Eq. (3), $c_{\mathrm{A} 0}$ symbolizes the initial concentration in mol/L of reactant $\mathrm{A}$ in the reaction mixture. Defining the concentration of reactant in a solvent-free melts as moles of reactant per volume of the reaction medium is problematic and impractical. To overcome this problem, the initial concentration of reactant A as a molar ratio between the initial moles of reactant A and total moles in initial reactant mixture was defined in Eq. (4). Based on the fact, that all experiments were performed with stoichiometric mixture of reactant A and B, the initial concentration of reactant $\mathrm{A}\left(c_{\mathrm{xA} 0}\right)$ can be described as (where $\mathrm{x}$ stands for the molar fraction): 


$$
c_{\mathrm{xA} 0}=0.5 \frac{\mathrm{mol}_{\mathrm{A}}}{\mathrm{mol}_{\mathrm{TOTAL}}}
$$

Also, due to the changed definition of the initial concentration defined in $\mathrm{mol}_{\mathrm{A}} / \mathrm{mol}_{\mathrm{TOTAL}}$, the rate of reaction in Eq. (4) is marked as $r_{\mathrm{A}}{ }^{*}$. Up to a certain conversion, the rate of reaction is chemically-controlled, which means that the rate of reaction at a particular reaction temperature depends predominantly on the activation energy of reaction and the pre-exponential factor, therefore:

$$
\begin{aligned}
& \left.k=k \quad \exp ^{(}-E_{\mathrm{A} 1}\right), \\
& { }_{k=k}^{1,0} \exp _{-}\left(\overline{E_{\mathrm{A} 2}} \dot{\bar{j}},\right. \\
& 2 \quad 2,0 \quad(\overline{R T})
\end{aligned}
$$

where $k_{1,0}$ and $k_{2,0}$ are pre-exponential factors of chemically-controlled DA and r-DA reaction, respectively, $E_{\mathrm{A} 1}$ activation energy of DA reaction $(\mathrm{J} / \mathrm{mol}), E_{\mathrm{A} 2}$ activation energy of $\mathrm{r}-\mathrm{DA}$ reaction $(\mathrm{J} / \mathrm{mol}), R$ ideal gas constant $(R$ $=8.314 \mathrm{~J} \mathrm{~mol}^{-1} \mathrm{~K}^{-1}$ ) and $T$ reaction temperature in Kelvin.

Experimental data obtained during the first 2 minutes of reaction at temperatures between $70{ }^{\circ} \mathrm{C}$ and $90{ }^{\circ} \mathrm{C}$ were used for estimating kinetic parameters of DA reaction. It was assumed that DA reaction in proposed timespan was only kinetically-controlled with the absence of the diffusion process. Moreover, there was insignificant influence of retro-Diels-Alder reaction at the beginning of the reaction due to the small concentration of formed product. Taking into account the two aforementioned assumptions, Eq. (4) can be simplified as:

$$
\left(-r_{A}^{*}\right)=\frac{d X_{\mathrm{A}}}{d t}=k c_{1 \mathrm{xA} 0}\left(1-X_{\mathrm{A}}\right)^{2} .
$$

As mentioned above, kinetic parameters of DA reaction were estimated by the least-square fitting of Eqs. (6) and (8) using experimental data obtained during the first 2 minutes of reaction at temperatures between $70{ }^{\circ} \mathrm{C}$ and 90 ${ }^{\circ} \mathrm{C}$. Determined kinetic parameters were $48.4 \mathrm{~kJ} \mathrm{~mol}^{-1}$ for activation energy $E_{\mathrm{A} 1}$ and $6.95 \cdot 10^{6} \mathrm{~mol}_{\mathrm{TOTAL}} \mathrm{mol}_{\mathrm{A}}{ }^{-1}$ $\min ^{-1}$ for $k_{1,0}$

Based on the rheological data, it was expected that the influence of diffusion is negligible at the reaction temperature of 100 and $110{ }^{\circ} \mathrm{C}$, which is why only DA and r-DA reaction effects were expected. In order to estimate kinetic parameters of r-DA reaction, the kinetic model taking into account DA and r-DA reaction (Eq. 4) was applied to experimental data with the least-square fitting method in this temperature range. Kinetic parameters of DA reaction, which were already estimated before, were kept constant. This procedure yielded the following kinetic parameters of r-DA reaction: $102.3 \mathrm{~kJ} \mathrm{~mol}^{-1}$ for activation energy $E_{\mathrm{A} 2}$ and $2.32 \cdot 10^{13} \mathrm{~min}^{-1}$ for $\mathrm{k}_{2,0}$. 
Štirn et al. [12] studied the Diels-Alder reaction between PMI and G-f in solution and found similar value for activation energies of DA and r-DA reaction, $48.4 \mathrm{~kJ} \mathrm{~mol}^{-1}$ and $102.3 \mathrm{~kJ} \mathrm{~mol}^{-1}$, respectively, whereas preexponential factors were estimated as $k_{1,0}=4.28 \cdot 10^{5} \mathrm{~L} \mathrm{~mol}^{-1} \mathrm{~min}^{-1}$ and $k_{2,0}=5.13 \cdot 10^{11} \mathrm{~min}^{-1}$. By comparing the kinetic parameters obtained in this study with those determined by Štirn [12], a minimal change in activation energy could be observed, when the reaction was performed in solution or in the melt. This confirms the assumption that the reaction mechanism does not change with the reaction medium. Based on the collision theory [30], the pre-exponential factor in Arrhenius equation presents a constant defining the rate of the reaction as a result of the frequency of collisions in the correct orientation. A higher number of successful collisions are therefore achieved by increasing the concentration of the reactant particles or raising the reaction temperature, which correspondingly leads to the increased rate of reaction. Unfortunately, pre-exponential factors of DA reaction in solution and melt cannot be compared due to the different units. Nevertheless, according to the collision theory, the pre-exponential factor of DA reaction in the melt is expected to be greater compared to the one in solution, due to the higher density of the reaction system.

\section{3. 1. Crossover between chemically and diffusion-controlled reaction kinetics}

In the development of the crossover between chemically and diffusion-controlled reaction kinetics, it was presumed that r-DA reaction is not influenced by the increase of reaction medium viscosity since it follows firstorder kinetics and there is no need for two reactants approach.

During the progress of the reaction in the melt, the viscosity of the reaction medium increased (Figure 4) leading to a point where the rate of reaction was no longer controlled by the intrinsic reaction kinetics but by the rate of reactants' approach. The effect of diffusion on the rate of reaction and consequential transition from chemicallycontrolled to diffusion-controlled reaction kinetics can be introduced to the kinetic model of DA reaction with Rabinowitch equation [29], which was derived from the activated complex theory. Based on the mentioned theory, the two reactive components diffuse toward each other to form an activated complex with possible creation of a stable bond. The reaction is controlled by diffusion dependent on the relative time the reactants need for the diffusion ( $\left.t_{\text {diffusion }}\right)$ and the time to form a chemical bond between the reactant molecules $\left(t_{\text {chem. kin. }}\right)($ Eq. (9)):

$$
t_{\text {reaction }}=t_{\text {diffusion }}+t_{\text {chem.kin. }}
$$

Eq. 8 can also be written in a form of kinetic parameters (Eq. (10)):

$$
\frac{1}{k_{\text {eff }}}=\frac{1}{k_{\mathrm{D}}}+\frac{1}{k_{\text {kin }}}
$$


where $t_{\text {reaction }}$ stands for the entire time needed for the reaction, $k_{\mathrm{D}}$ is the diffusion rate constant, $k_{\text {eff }}$ effective rate constant and $k_{\text {kin }}$ intrinsic chemical reaction rate constant. Although Rabinowitch [29] derived Eq. (10) for diffusion-controlled kinetics of a small-molecule reaction, it has been further used in a number of studies involving reactions in different systems, including polymers [31, 32].

Diffusion-controlled reactions have been the subject of significant studies and several models have been proposed to relate the rate constant to appropriate diffusion constant [33]. Among the proposed diffusion controlled models, the most frequently used correlation between mutual diffusion coefficient of reactants $\left(D_{\mathrm{AB}}\right)$ and diffusion rate constant is the Smoluchowski equation [34], which was derived from diffusion-controlled reactions in solutions (Eq. (11)):

$$
k_{\mathrm{D}}=4 \pi r_{\mathrm{AB}} D_{\mathrm{AB}}
$$

where $r_{\mathrm{AB}}$ stands for encounter distance, which is the distance between reactants $\mathrm{A}$ and $\mathrm{B}$ necessary for the chemical transformation. Furthermore, diffusion coefficient $D_{\mathrm{AB}}$ could be estimated by the Stokes-Einstein expression [35] (Eq. (12)), where the viscosity effect of reaction medium is also incorporated:

$$
D_{\mathrm{AB}}=\frac{k_{\mathrm{B}} T}{\pi \beta \mu r_{\mathrm{s}}}
$$

where $\mu$ is viscosity of the medium, $r_{s}$ hydrodynamic radius of the molecule and $\beta$ a numerical constant. The combination of equations (9)-(12) shows the effect of increasing viscosity of the melt on reaction kinetics. The majority of models, describing the transition from chemically to diffusion-controlled kinetics, are semi-empirical, since mechanistic models would involve many complex steps and a large number of adjustable parameters. A semi-empirical model based on the free-volume theory was developed by Marten and Hamielec [24] for high conversion diffusion-controlled polymerization of styrene. When the free volume of reaction system $v_{\mathrm{f}}$, which is related to conversion, reaches a critical value $v_{\mathrm{f}, \mathrm{cr}}$, diffusion becomes controlling and the diffusion rate constant is given by:

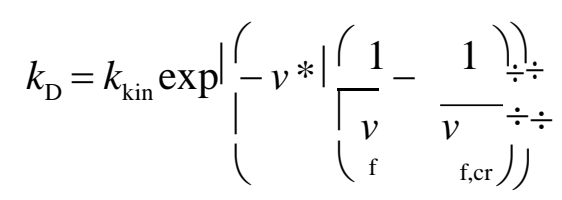

where $v^{*}$ is a constant which depends on the temperature and the reaction system. Another, more fundamental chemical-diffusion model was proposed by Cook [20] for the study case of epoxy-amino resins curing. The model is based on the free-volume concept and similar to the Williams-Landel-Ferry (WLF) equation [36]. Vyazovkin and Sbirrazzuoli [37] defined diffusion rate constant with separable contributions of temperature and conversion: 


$$
k_{\mathrm{D}}=D_{0} \exp \left({\frac{E_{\mathrm{D}}}{R T}}^{+} B X_{\dot{\bar{j}}}\right)
$$

where $D_{0}$ is the pre-exponential factor, $E_{\mathrm{D}}$ the activation energy of diffusion, $X$ conversion of reactants and $B$ a constant that accounts for the change in the conditions of diffusion due to the progress of the reaction. In addition to semi-empirical models based on the free-volume concept, the loss of mobility due to vitrification can also be quantified by a mobility factor defined by normalizing the heat capacities, which are measured with modulated temperature differential scanning calorimetry (MTDSC) [38].

Instead of using the previously described definition of the diffusion-controlled kinetic parameter, the semiempirical model developed by Marten and Hamielec [24] was used in this study. The aforementioned model proposed by Chern and Poehlein [21] was adjusted by introducing important assumptions in the derivation of Eq. (13) for the purpose of validating the use of the model to describe the DA reaction in the molten state. During the derivation of Eq. (13), authors assumed that the rate of the reaction becomes influenced by the diffusion of reactants when the mutual diffusion coefficient $\left(D_{\mathrm{AB}}\right)$ of reactants becomes equal or lower than the critical value of diffusion coefficient $\left(D_{\mathrm{AB}, \mathrm{cr}}\right)$ :

$$
D_{\mathrm{AB}} \leq D_{\mathrm{AB}, \mathrm{cr}}
$$

The rate of diffusion is defined as:

$$
k_{\mathrm{D}}=K(T) D_{\mathrm{AB}}
$$

where $K(T)$ is an unknown temperature dependent constant. The variation of mutual diffusion coefficient of reactants during the progress of reaction is described with the help of the correlation proposed by Bueche [39], which is based on the free-volume theory and relates the diffusion coefficient of species $i\left(D_{\mathrm{i}}\right)$ to the free volume of the reaction medium $\left(v_{\mathrm{f}}\right)$ :

$$
D_{\mathrm{i}}=\frac{\phi_{0} \delta^{2}}{6} \exp \left(-\frac{\left.\beta^{*} v^{*}\right)}{\left.v_{f}\right)} \div\right.
$$

where $\phi_{0}$ stands for jump frequency of molecules at high temperatures, $\delta$ is jump distance or average change in position of the centre of the mass resulting from such jump, $\beta^{*}$ a numerical constant, $v^{*}$ a critical amount of free volume per molecule for a jump to another position to be realized and $v_{f}$ an average amount of free volume assigned to a particular molecule. A more exact derivation of Eq. (17) can be found elsewhere [39]. The transition from chemically-controlled to diffusion-controlled reaction kinetics is caused by a decrease in the free volume available to the molecular motion below a certain value. 
Chern and Poehlein [21] modified Eq. (13) with the aim of describing condensation polymerization. They argued that the free volume per molecule $\left(v_{\mathrm{f}}\right)$ is highly related to the conversion of reactants $\left(X_{\mathrm{A}}\right)$ and proposed the following correlation:

$$
\frac{v^{*}}{v_{f}}=A_{0}+A_{1} X_{\mathrm{A}}
$$

where $A_{0}$ and $A_{1}$ are numerical constants dependent on the reaction system and temperature. With the application of Eq. (17) to Eq. (12) the following equation can be obtained:

$$
k_{\mathrm{D}}=k_{\mathrm{kin}} \exp \left(-C\left(X_{\mathrm{A}}-X_{\mathrm{c}}\right)\right)
$$

where $X_{\mathrm{c}}$ is a critical conversion, when the rate of reaction becomes restricted by the mobility of reactants and $C$ is an empirical constant that depends on the structure, system, and temperature. Eq. (19) means an abrupt transition from the diffusion to chemical control of the reaction after the conversion reaches the critical value, $X_{\mathrm{c}}$. However, this change is gradual and there is a region where both factors control the rate of reaction. The overall effective

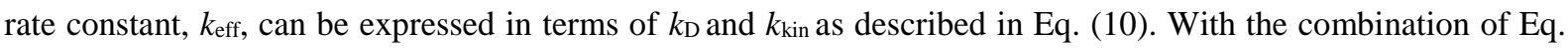
(4), (10) and (19) the kinetic model used to describe Diels-Alder reaction in the molten state was derived:

$$
\left(-r^{*}\right)=\frac{d X_{\mathrm{A}}}{d t}=\frac{k_{1}}{1+\exp \left(C\left(X_{\mathrm{A}}-X_{\mathrm{c}}\right)\right)} c{ }_{\mathrm{xA} 0}^{(1-X)^{2}-k X .}
$$

When $X_{\mathrm{A}}$ is much lower than the critical value, $X_{\mathrm{c}}$, the reaction is kinetically controlled and the effect of diffusion is negligible, hence Eq. (20) is reduced to Eq. (4). When $X_{\mathrm{A}}$ increases and approaches to $X_{\mathrm{c}}$, both factors, $k_{\mathrm{D}}$ and

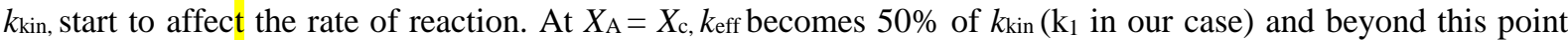
approaches zero as the reaction effectively stops. All authors agree that the critical conversion $X_{\mathrm{c}}$ increases with the increasing temperature [40-44]. The following trend was ascribed to the increased mobility of molecules due to higher temperature and consequentially delay in the effect of diffusion on the rate of reaction to higher conversions. On the other hand, there is a large disagreement in the literature regarding associating the variation of empirical constant $\mathrm{C}$ with the temperature. Some authors found $C$ to be temperature dependent [21], whereas others observed no discernible trend in the variation of $C$ with the reaction temperature [42-44]. If one observes just the first part of the Eq. (20), the effective rate constant as a function of temperature is:

$$
k_{\text {eff }}=\frac{k_{1}}{1+\exp \left(C\left(X_{\mathrm{A}}-X_{\mathrm{c}}\right)\right)} \text {, }
$$

where $X_{\mathrm{c}}$ describes an inflection point from a mathematical point of view, and the parameter $C$ defines steepness of the function around the inflection point, with higher values of $C$ leading to steeper function and vice versa. To 
put it differently, higher values of the parameter $C$ mean that the transition from chemically-controlled reaction kinetics to diffusion-controlled is faster and that the region where both, diffusion and chemical kinetics, control the rate of reaction is narrower. It is, therefore, argued that the rate of this transition, and thus the empirical constant $C$, cannot be a random function of temperature. Based on the isothermal rheological study (Figure 4) it was assumed that the parameter $C$ increases linearly with the reaction temperature. Parameters $X_{\mathrm{c}}$ and $C$ were, therefore, described as linear functions of reaction temperature:

$$
\begin{aligned}
& X_{\mathrm{c}}=a+b T \\
& C=d T+e
\end{aligned}
$$

In Eq. (21) $a, b, d, e$ are empirical parameters independent of temperature and $T$ reaction temperature in ${ }^{\circ} \mathrm{C}$.

\section{3. 2. Kinetic model development and determination of kinetic parameters}

Equations (6), (20) and (22) present a kinetic model of DA reaction in a molten state, which incorporates effects of DA and r-DA reaction on the progress of the reaction in addition to the crossover between chemically and diffusion-controlled reaction kinetics of DA reaction. Based on the previous experimental and fitting findings, the values of activation energies for DA $\left(E_{\mathrm{A} 1}\right)$ and r-DA reaction $\left(E_{\mathrm{A} 2}\right)$ were used and remained constant during the fitting procedure: $E_{\mathrm{A} 1}=48.4 \mathrm{~kJ} \mathrm{~mol}^{-1}$ and $E_{\mathrm{A} 2}=102.3 \mathrm{~kJ} \mathrm{~mol}^{-1}$. Additionally, the values of pre-exponential factors, namely $k_{1,0}=6.95 \cdot 10^{6} \mathrm{~mol}_{\mathrm{TOTAL}} \mathrm{mol}_{\mathrm{A}}^{-1} \mathrm{~min}^{-1}$ and $k_{2,0}=2.32 \cdot 10^{13} \mathrm{~min}^{-1}$, were used as the first approximation of parameters and a small variation was allowed. In order to estimate unknown parameters in Eqs. (6), (20) and (22), the method of the least-square fitting of the aforementioned equations on the experimental data was used. The following relations for temperature-dependence of parameters $X_{\mathrm{c}}$ and $C$ were obtained:

$$
\begin{aligned}
& X_{C}=0.4185+0.0050 \times T \\
& C=1.293 \times T-73.69
\end{aligned}
$$

Since a small variation of pre-exponential factors of DA and r-DA reaction was allowed, the procedure yielded a value $7.24 \cdot 10^{6} \mathrm{~mol}_{\mathrm{TOTAL}} \mathrm{mol}_{\mathrm{A}}^{-1} \mathrm{~min}^{-1}$ for $k_{1,0}$ and $2.34 \cdot 10^{13} \mathrm{~min}^{-1}$ for $k_{2,0}$. By comparing those values to the values obtained in the previous section, it can be concluded that assumptions made during the first estimation of kinetic parameters are confirmed since the final values of kinetic parameters are truly close to those previously obtained. By applying the kinetic model (Eq. 20), a satisfying agreement between the experimental data and the kinetic model was achieved (Figure 5). The parity plot presented in Figure 6 shows the comparison between the calculated values with those determined experimentally. The trend between calculated and experimental data is the same and is within the $90 \%$ confidence interval. Slight deviation at the temperature $70{ }^{\circ} \mathrm{C}$ could be observed in the first 3 
minutes of the reaction, where a higher degree of conversion was predicted by the model, which could be associated with a minor delay of melting effects of the mixture. Table 1 shows the values of $X_{\mathrm{c}}$ and $C$ at different reaction temperatures. For better representation, the Table 1 shows the values of maximum conversion obtained after prolonged heating (90 min). Comparing values of critical and maximum conversion surprisingly show, the relatively high values of critical conversion, $X_{\mathrm{c}}$. It has to be emphasized that $X_{\mathrm{c}}$ does not represent the value of conversion when diffusion starts to influence the rate of reaction, but rather he value at which $k_{\text {eff }}$ becomes $50 \%$

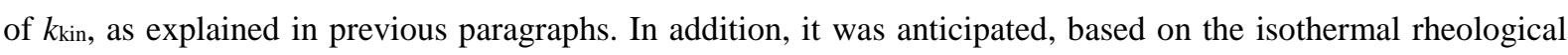
data (Figure 4), that diffusion has an evident impact on the progress of reaction especially at the lowest two temperatures, $70{ }^{\circ} \mathrm{C}$ and $80^{\circ} \mathrm{C}$. The same behaviour was predicted by the presented kinetic model.

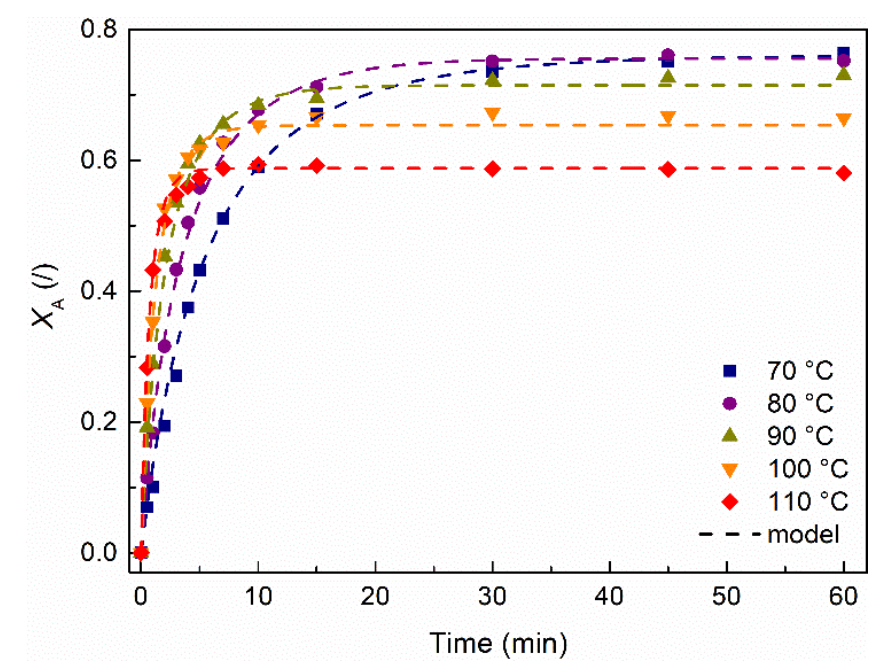

Fig. 5 Comparison between the experimental data for progress of reaction at different reaction temperatures and the progress of the reaction predicted by presented kinetic model of DA reaction in molten state.

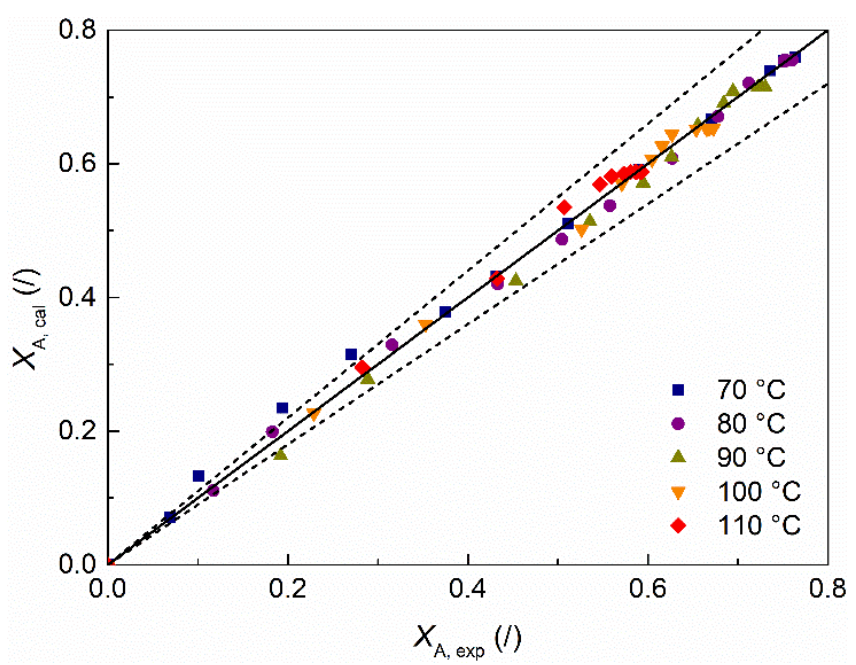

Fig. 6 Comparison of the calculated conversions (y-axis) and experimentally determined conversions (x-axis) of the studied reaction with $90 \%$ confidence intervals. 
Table 1 Values of diffusion-controlled reaction parameters $X_{\mathrm{c}}$ and $C$ at different reaction temperatures $(T)$ compared to the maximum conversion $\left(X_{\max }\right)$ obtained after prolonged heating (90 min).

\begin{tabular}{cccc}
\hline $\boldsymbol{T}\left({ }^{\circ} \mathbf{C}\right)$ & $\boldsymbol{X}_{\max }(/)$ & $\boldsymbol{X}_{\mathbf{c}}(/)$ & $\boldsymbol{C}(/)$ \\
\hline 70 & 0.81 & 0.77 & 16.8 \\
80 & 0.80 & 0.82 & 29.8 \\
90 & 0.76 & 0.87 & 42.7 \\
100 & 0.67 & 0.92 & 55.6 \\
110 & 0.59 & 0.97 & 68.6
\end{tabular}

A phenomenological kinetic model has been proposed with which it is possible to describe the rate of reaction of the investigated reaction system in the molten state over the whole course of the reaction for reaction temperatures from $70{ }^{\circ} \mathrm{C}$ to $110{ }^{\circ} \mathrm{C}$. The model is based on the combination of Rabinowitch equation [29] and the model proposed by Chern and Pohlein [21] and describes the crossover between activation and diffusion controlled DielsAlder reaction with the included contribution of retro-Diels-Alder reaction.

\section{Conclusions}

To conclude, this study showed a detailed investigation of thermal and viscoelastic behaviour during the DA reaction and provided crucial new knowledge for application of self-healing ability to benzoxazine-based thermosetting resins. The processes that occurred in the system were identified by non-isothermal DSC and rheological analysis performed in the same temperature range. Rheological data proved to be of significant value in the kinetic model development and interpretation. The DA reaction between PMI and G-f resulted in an increase of a melt's viscosity for a few orders of magnitude. The kinetics of DA reaction was studied by isothermal heating of PMI and G-f mixture accompanied by ${ }^{1} \mathrm{H}$ NMR analysis to determine the conversion of reactants. Interestingly, the maximum conversion of isothermal experiments did not vary significantly with increased reaction temperature and never reached the value of unity. Further investigation was, therefore, designed to shed light on the processes causing the reaction rate limitation and consequently effect the incomplete conversion of reactants. After a comprehensive examination of the studied system, the crossover between chemically and diffusion-controlled reaction due to the increasing viscosity of reaction system was identified as a main reason for decrease in the reaction rate. By taking into account intrinsic reaction kinetics of Diels-Alder reaction between PMI and G-f and 
the effect of the reaction medium, the kinetic model based on the Rabinowitch equation and Chern-Poehlein model was developed.

\section{Acknowledgements}

The financial support of this work by the Slovenian Research Agency (Grant P2-0191 and grant N2-0033) is gratefully acknowledged. 


\section{References}

1. Nicolaou KC, Snyder SA, Montagnon T, Vassilikogiannakis G (2002) The Diels-Alder reaction in total synthesis. Angew Chemie - Int Ed 41:1668-1698. https://doi.org/10.1002/15213773(20020517)41:10<1668::AID-ANIE1668>3.0.CO;2-Z

2. Martin JG, Hill RK (1961) Stereochemistry of the Diels-Alder Reaction. Chem Rev 61:537-562. https://doi.org/10.1021/cr60214a001

3. Kolb HC, Finn MG, Sharpless KB (2001) Click chemistry: Diverse chemical function from a few good reactions. Angew Chemie - Int Ed 40:2004-2021. https://doi.org/10.1002/15213773(20010601)40:11<2004::AID-ANIE2004>3.3.CO;2-X

4. Gandini A (2013) The furan/maleimide Diels-Alder reaction: A versatile click-unclick tool in macromolecular synthesis. Prog Polym Sci 38:1-29.https://doi.org/10.1016/j.progpolymsci.2012.04.002

5. Liu Y-L, Chuo T-W (2013) Self-healing polymers based on thermally reversible Diels-Alderchemistry. Polym Chem 4:2194. https://doi.org/10.1039/c2py20957h

6. Kötteritzsch J, Hager MD, Schubert US (2015) Tuning the self-healing behavior of one-component intrinsic polymers. Polymer (Guildf) 69:321-329. https://doi.org/10.1016/j.polymer.2015.03.027

7. Rao BS, Palanisamy A (2013) Synthesis of bio based low temperature curable liquid epoxy, benzoxazine monomer system from cardanol: Thermal and viscoelastic properties. Eur Polym J 49:2365-2376. https://doi.org/10.1016/j.eurpolymj.2013.05.029

8. Calo E, Maffezzoli A, Mele G, et al (2007) Synthesis of a novel cardanol-based benzoxazine monomer and environmentally sustainable production of polymers and bio-composites. Green Chem 9:754-759. https://doi.org/10.1039/b617180j

9. Gandini A, Coelho D, Silvestre AJD (2008) Reversible click chemistry at the service of macromolecular materials. Part 1: Kinetics of the Diels-Alder reaction applied to furan-maleimide model compounds and linear polymerizations. Eur Polym J 44:4029-4036.

https://doi.org/10.1016/J.EURPOLYMJ.2008.09.026

10. Tian Q, Yuan YC, Rong MZ, Zhang MQ (2009) A thermally remendable epoxy resin. J Mater Chem 19:1289-1296. https://doi.org/10.1039/b811938d

11. Bose RK, Kötteritzsch J, Garcia SJ, et al (2014) A rheological and spectroscopic study on the kinetics of self-healing in a single-component diels-alder copolymer and its underlying chemical reaction. J Polym Sci Part A Polym Chem 52:1669-1675. https://doi.org/10.1002/pola.27164 
12. Štirn Ž, Ručigaj A, Krajnc M (2016) Characterization and kinetic study of Diels-Alder reaction: Detailed study on $\mathrm{N}$-phenylmaleimide and furan based benzoxazine with potential self-healing application.

Express Polym Lett 10:537-547. https://doi.org/10.3144/expresspolymlett.2016.51

13. Magana S, Zerroukhi A, Jegat C, Mignard N (2010) Thermally reversible crosslinked polyethylene using Diels-Alder reaction in molten state. React Funct Polym 70:442-448.

https://doi.org/10.1016/j.reactfunctpolym.2010.04.007

14. Jegat C, Mignard N (2008) Effect of the polymer matrix on the thermal behaviour of a furan-maleimide type adduct in the molten state. Polym Bull 60:799-808.https://doi.org/10.1007/s00289-008-0913-y

15. Bala MD, Coville NJ (2007) Organometallic chemistry in the melt phase. J Organomet Chem 692:709730. https://doi.org/10.1016/j.jorganchem.2006.10.046

16. Rothenberg G, Downie AP, Raston CL, Scott JL (2001) Understanding solid/solid organic reactions. J Am Chem Soc 123:8701-8708. https://doi.org/10.1021/ja0034388

17. Galwey AK (1994) Thermal reactions of selected solids including reactants that melt during chemical change. J Therm Anal 41:267-286. https://doi.org/10.1007/BF02549315

18. Galwey AK (1995) Crystal chemistry and molecular mechanisms in molten magmas: the significance of fusion in reactions of solids. Thermochim Acta 269-270:621-630. https://doi.org/10.1016/0040-

$$
\text { 6031(95)02355-0 }
$$

19. Anastas PT, Warner JC (1998) Green chemistry: theory and practice. Oxford University Press, New York

20. Wise CW, Cook WD, Goodwin AA (1997) Chemico-diffusion kinetics epoxy-amine resins of model. Polymer (Guildf) 38:3251-3261. https://doi.org/10.1016/S0032-3861(96)00882-8

21. Chern CS, Poehlein GW (1987) A kinetic model for curing reactions of epoxides with amines. Polym Eng Sci 27:788-795. https://doi.org/10.1002/pen.760271104

22. Fournier J, Williams G, Duch C, Aldridge GA (1996) Changes in molecular dynamics during bulk polymerization of an epoxide-amine system as studied by dielectric relaxation spectroscopy. Macromolecules 29:7097-7107. https://doi.org/10.1021/ma9517862

23. Cole KC (1991) A new approach to modeling the cure kinetics of epoxy amine thermosetting resins. 1. Mathematical development. Macromolecules 24:3093-3097. https://doi.org/10.1021/ma00011a011

24. Marten FL, Hamielec AE (1979) High conversion diffusion-controlled polymerization. In: Henderson JN, Bouton CT (eds) Polymerization Reactors and Processes. ACS Symposium Series, Washington, DC, 
pp 43-70

25. Sundberg DC, Hsieh JY, Soh SK, Baldus RF (1981) Diffusion-controlled kinetics in the emulsion polymerization of styrene and methyl methacrylate. In: Basset RD, Hamielec EA (eds) Emulsion Polymers and Emulsion Polymerization, 165th ed. ACS Symposium Series, Washington, DC, pp 327343

26. Wang C, Sun J, Liu X, et al (2012) Synthesis and copolymerization of fully bio-based benzoxazines from guaiacol, furfurylamine and stearylamine. Green Chem 14:2799-2806. https://doi.org/10.1039/c2gc35796h

27. Canadell J, Fischer H, De With G, van Benthem RATM (2010) Stereoisomeric effects in thermoremendable polymer networks based on Diels-Alder crosslink reactions. J Polym Sci Part A Polym Chem 48:3456-3467. https://doi.org/10.1002/pola.24134

28. Galwey AK, Brown ME (1998) Chapter 3 - Kinetic background to thermal analysis and calorimetry. In: Handbook of Thermal Analysis and Calorimetry. Elsevier Science B.V., Amsterdam, pp 147-224

29. Rabinowitch E (1937) Collision, co-ordination, diffusion and reaction velocity in condensed systems. Trans Faraday Soc 33:1225-1233. https://doi.org/10.1039/tf9373301225

30. Atkins PW, De Paula J (2010) Atkins' Physical chemistry, 9th ed. Oxford University Press, Oxford

31. Deng Y, Martin GC (1994) Diffusion and diffusion-controlled kinetics during epoxy-amine cure. Macromolecules 27:5147-5153. https://doi.org/10.1021/ma00096a043

32. Huguenin FGAE, Klein MT (1985) Intrinsic and transport-limited epoxyamine cure kinetics. Ind Eng Chem Prod Res Dev 24:166-171. https://doi.org/10.1021/i300017a031

33. Allen PEM, Patrick CR (1974) Kinetics and mechanisms of polymerization reactions: Application of physicochemical principles. E. Horwood, Chichester, New York

34. Bamford CH, Tipper CFH, Compton RG (1985) Chapter 2 Diffusion-Controlled Reactions in Solution. In: Comprehensive Chemical Kinetics. Elsevier, pp 3-46

35. Becker SR, Poole PH, Starr FW (2006) Fractional Stokes-Einstein and Debye-Stokes-Einstein relations in a network-forming liquid. Phys Rev Lett 97:055901.https://doi.org/10.1103/PhysRevLett.97.055901

36. Williams ML, Landel RF, Ferry JD (1955) The temperature dependence of relaxation mechanisms in amorphous polymers and other glass-forming liquids. J Am Chem Soc 77:3701-3707.

https://doi.org/10.1021/ja01619a008

37. Vyazovkin S, Sbirrazzuoli N (1996) Mechanism and kinetics of epoxy-amine cure studied by 
differential scanning calorimetry. Macromolecules 29:1867-1873. https://doi.org/10.1021/ma951162w

38. Van Assche G, Swier S, Van Mele B (2002) Modeling and experimental verification of the kinetics of reacting polymer systems. Thermochim Acta 388:327-341. https://doi.org/10.1016/S00406031(02)00038-2

39. Bueche FJ (1982) Physical properties of polymers. Interscience Publishers, New York

40. Du S, Guo ZS, Zhang B, Wu Z (2004) Cure kinetics of epoxy resin used for advanced composites. Polym Int 53:1343-1347. https://doi.org/10.1002/pi.1533

41. Liu G, Zhao B, Zhou X, et al (2006) Curing kinetics of diglycidyl ether of bisphenol a and diaminodiphenylmethane using a mechanistic model. Macromol Theory Simulations 15:339-346. https://doi.org/10.1002/mats.200500060

42. Karayannidou EG, Achilias DS, Sideridou ID (2006) Cure kinetics of epoxy-amine resins used in the restoration of works of art from glass or ceramic. Eur Polym J 42:3311-3323.

https://doi.org/10.1016/j.eurpolymj.2006.08.025

43. Kim WG, Lee JY (2002) Curing characteristics of epoxy resin systems that include a biphenyl moiety. J Appl Polym Sci 86:1942-1952. https://doi.org/10.1002/app.11104

44. Cole KC, Hechler JJ, Noël D (1991) A new approach to modeling the cure kinetics of epoxy amine thermosetting resins. 2. Application to a typical system based on bis[4-(diglycidylamino)phenyl]methane and Bis(4-aminophenyl) sulfone. Macromolecules 24:3098-3110.https://doi.org/10.1021/ma00011a012 